\title{
The impact of intrauterine and extrauterine weight gain in premature infants on later body composition
}

\author{
Miguel Saenz de Pipaon ${ }^{1}$, Izaskun Dorronsoro ${ }^{1}$, Laura Álvarez-Cuervo ${ }^{1}$, Nancy F. Butte ${ }^{2}$, Rosario Madero ${ }^{3}$, \\ Vicente Barrios ${ }^{4}$, Juan Coya ${ }^{5}$, Miriam Martínez-Biarge ${ }^{1}$, Gabriel Á. Martos-Moreno ${ }^{4}$, Mary S. Fewtrell ${ }^{6}$, Jesús Argente ${ }^{4}$ and \\ José Quero ${ }^{1}$
}

\begin{abstract}
BACKGROUND: The impact of intrauterine and extrauterine growth on later insulin resistance and fat mass (FM) in very low birth weight (VLBW) infants is not well established. The aim of our study was to evaluate the effects of intrauterine and early/late extrauterine growth on later insulin resistance and body composition in VLBW infants from 6 months' corrected age (CA) to 36 months.
\end{abstract}

METHODS: Prospective measurements of body composition by dual-energy $\mathrm{X}$-ray absorptiometry and insulin resistance by homeostasis model assessment insulin resistance (HOMA-IR) along with other fasting plasma biochemistries were made in 95 VLBW infants at 6, 12, 18, and 24 months' CA and 36 months' postnatal age. Mixed-effect models were used to evaluate the effects of age, sex, maturation status, and $\Delta$ weight SD score on percentage FM (PFM), FM index (FMI), fat-free mass index (FFMI), and HOMA-IR.

RESULTS: PFM and FMI were negatively associated with a decrease in weight-SD scores from birth to 36 weeks' postmenstrual age $(P M A ; P=0.001)$ and from 36 weeks' PMA to 6 months' $C A(P=0.003)$. PFM and FMI were higher in AGA than in small for gestational age (SGA) infants. HOMA-IR was not associated with the $\Delta$ weight-SD scores in either period. CONCLUSIONS: Catch-down growth in terms of weight is associated with persistently lower adiposity but not insulin resistance up to 36 months of age.

$\mathbf{T}$ he effects of prematurity, early growth impairment, and catch-up growth of premature infants on their later metabolic health and adiposity remain unresolved. Although premature birth is associated with a reduction in insulin sensitivity in childhood $(1,2)$, the effects of early growth patterns of preterm infants on insulin sensitivity are inconclusive. In two studies in preterm infants, associations between rapid weight gain in the first 2 weeks of life and decreased insulin sensitivity were observed in adolescents and prepubertal children $(3,4)$, although the fasting $32-33$ split proinsulin concentration of formerly preterm infants with greater weight gain was not statistically different from that of controls during adolescence (3). Rapid infant weight gain until 3 months' corrected age (CA) is reported to be associated with higher HOMA-IR at 19 years and marginally so at 5-7 years; however, the association was not statistically significant after correction for possible confounding variables $(4,5)$. Other studies failed to show any effect of growth between birth and 18 months of age on insulin concentrations at 9-12 years of age $(6,7)$.

The early postnatal period is purported to be critical for the development of adipose tissue (8). Preterm infants have a greater percentage fat mass (PFM) at term gestational age than full-term infants (9). Rapid growth in the first few months of life in very low birth weight (VLBW) infants has been associated with increased adiposity at 6 and 19 years of age (10-12). In contrast, prematurity was associated with reduced body fatness in later childhood in one study (13), but with greater FM and trunk fat at 18-24 years of age compared with full-term subjects in another study (14).

The effect of intrauterine weight gain in premature infants on later insulin sensitivity is unclear. Although size at birth influences plasma glucose levels at 60 and $30 \mathrm{~min}$ after a glucose load in formerly preterm children at 20 days and 9-12 years, respectively $(6,15)$, it did not affect insulin sensitivity at 7 days of age or in adulthood $(7,16,17)$. We previously reported higher HOMA-IR in the cord blood of preterm infants, and a negative correlation between HOMA-IR and insulin levels and gestational age in this cohort (18).

To address these unresolved issues, our study aims were as follows: (i) to determine the effect of intrauterine and early/ late extrauterine growth on later insulin resistance and body composition from 6 months' CA to 36 months; (ii) to analyze the concentrations of fasting plasma triglycerides, cholesterol, leptin, adiponectin, resistin, and insulin-like growth factor I

\footnotetext{
${ }^{1}$ Department of Neonatology, Hospital Universitario La Paz, Red de Salud Materno Infantil y Desarrollo - SAMID, Universidad Autónoma de Madrid, Madrid, Spain; ${ }^{2}$ USDA/ARS Children's Nutrition Research Center, Pediatrics, Baylor College of Medicine, Houston, Texas, USA; ${ }^{3}$ Department of Biostatistics, Hospital Universitario La Paz, Madrid, Spain; ${ }^{4}$ Department of Pediatrics and Endocrinology, Hospital Infantil Universitario Niño Jesús, Instituto de Investigación La Princesa, Department of Pediatrics, CIBER Fisiopatología de la Obesidad y Nutrición (CIBEROBN), IMDEA Food Institute, Universidad Autónoma de Madrid, Madrid, Spain; ${ }^{5}$ Department of Nuclear Medicine, Hospital Universitario La Paz, Madrid, Spain; ${ }^{6}$ Childhood Nutrition Research Centre, UCL Institute of Child Health, London, UK. Correspondence: Miguel Saenz de Pipaon (miguel.saenz@salud.madrid.org) 
from 6 months' CA to 36 months; and (iii) to test whether these fasting plasma biochemistries correlate with PFM and HOMA-IR in VLBW infants.

\section{METHODS}

A cohort study of VLBW was conducted between 6 and 36 months corrected for gestational age. Infants were recruited from $\mathrm{La} \mathrm{Paz}$ University Hospital between November 2004 and October 2006 immediately after birth. Among all consecutive infants $(n=341)$ who were born at $<34$ weeks' gestation and with birth weight $<1,500 \mathrm{~g}$, parents of 111 infants gave written informed consent to participate. Exclusion criteria included presence of congenital diseases, chromosomal abnormalities, and short bowel syndrome or other digestive disorders in which absorption of nutrients was impaired. Data from VLBW infants were collected during initial admission.

The local research ethics committee of the La Paz University Hospital approved the study, and written informed consent was obtained from the parent(s).

Recombinant human erythropoietin and a supplement of elemental iron $(6 \mathrm{mg} / \mathrm{kg}$ per day) from the first few days of life were administered to all VLBW infants included in the cohort during hospital stay. The early nutrition and clinical course of the premature infants were described previously (19). Data regarding human milk duration or formula feeding were collected. Weight $(\mathrm{g})$, length $(\mathrm{cm})$, and head circumference $(\mathrm{cm})$ were measured at birth and at 36 weeks' PMA, and they were expressed as SD scores to correct for gestational age and sex with the use of Fenton growth chart (20). Infants were classified as AGA or SGA at birth regarding weight for age above or below 10th percentile.

Nutritional management after discharge of the VLBW infants entailed the following recommendations: (i) a supplement of elemental iron at $2 \mathrm{mg} / \mathrm{kg}$ per day starting at the age of 2 months and extending through 12 months of age; (ii) exclusive breastfeeding for a minimum of 4 months but preferably for 6 months, without supplementation after discharge; and (iii) if human milk was not available, post-discharge formula was used until 3 months' CA and preterm formula was used only for infants with bronchopulmonary dysplasia. The iron requirements for toddlers (1-3 years of age) were presumed to be met with naturally iron-enriched foods.

For the research protocol, infants were scheduled a visit to the La Paz University Hospital at 6, 12, 18, and 24 months' CA and at 36 months. Two physicians performed anthropometric measures that included weight, length, and head circumference according to standardized procedures. During the study, standardization was carried out to maximize interobserver reliability, and coefficient of variations less than $5 \%$ was achieved and maintained during the study period. A nude weight was obtained in duplicate using a Seca electronic infant scale accurate to the nearest $10 \mathrm{~g}$ (Seca 375, Hamburg, Germany), and length was obtained in duplicate on an infant length board to the nearest $0.1 \mathrm{~cm}$ (Seca 210). Circumference measurement was taken to the nearest $\mathrm{mm}$ by using a flexible measuring tape. Whole-body fat mass (FM), fat-free mass (FFM), and regional (truncal) FM, whole-body bone mineral content, and bone mineral density (BMD) were measured by using dual X-ray absorptiometry (Lunar- DPX-MD; GE Healthcare, Chalfont, St Giles, UK). For the measurement of truncal fat, a line of delineation was drawn between the head of the humerus and the glenoid fossa of the scapula to separate the upper limb from the trunk, and the leg consisted of the parts of the body between the inferior border of the ischial tuberosity to the most distal tip of the toes.

Scans were analyzed using infant whole-body analysis software (General Electric, Chalfont, St. Giles, UK). All dual X-ray absorptiometry scans were performed with the same device and software. No sedation was used. The study was performed after a feeding. Previously reported precision values for dual X-ray absorptiometry are $<1 \%$ for FFM and $<2 \%$ for FM (21). FM was normalized to body weight using the FMP, and FM and FFM were normalized to body height to give fat mass index (FMI) and fat-free mass index (FFMI) as follows: $\mathrm{FMI}=\mathrm{FM} /$ height $^{2}$ and $\mathrm{FFMI}=\mathrm{FFM} /$ height $^{2}$. The percentage of truncal fat (PTF) was calculated using the following formula: PTF $=100 \times \mathrm{TF} / \mathrm{FM}$.

Blood samples were drawn under fasting conditions, at least $6 \mathrm{~h}$ after the previous meal, centrifuged, and the serum stored at $-80^{\circ} \mathrm{C}$ until being assayed. Glucose, total cholesterol, and triglycerides were immediately quantified by enzymatic methods in an autoanalyzer. Levels of insulin (Diagnostic Products Corporation, Los Angeles, CA), leptin, adiponectin, and insulin-like growth factor I (Mediagnost, Tübingen, Germany) were determined by radioimmunoassay and resistin by enzyme-linked immunosorbent assay from Merck Millipore (Billerica, MA). In all cases, intra- and inter-assay coefficients of variations were lower than 10\%. HOMA-IR was calculated according to the following formula: (glucose $(\mathrm{mmol} / \mathrm{l}) \times$ insulin $(\mathrm{mU} / \mathrm{ml})) / 22.5$.

At 6-36 months, weight, length, and head circumference $z$-scores were computed using OMS growth curves through the macro for SPSS. From 6 to 24 months, CA was used, and chronological age was used at 36 months. Extrauterine growth retardation between birth and 36 weeks' PMA (early) and between 36 weeks' PMA and 6 months' CA (late) was computed as $\Delta-$ SD scores. The number of participants completing the 6- to 36-month visits was 95; the parents of 16 infants refused to participate in the followup study.

The study was originally designed to evaluate the effect of early growth retardation on body composition and metabolic outcomes. Mixed-effect model repeat measurement analyses were performed to assess associations between prenatal (as a dichotomous variable, AGA vs. SGA), early and late extrauterine growth retardation (both continuous variables, changes in SDS), and PFM, FMI, FFMI, and HOMA-IR, from 6 to 36 months, using the MIXED procedure for the SAS 9.1 software (SAS Institute, Cary, NC). The models included AGA-SGA at birth, the age at evaluation $(6,12,18,24$, and 36 months), and early and late $\Delta-\mathrm{SD}$ scores. Sex was used as a covariate. An estimate of fixed effect was calculated for PFM, FMI, FFMI, and HOMA-IR regarding prenatal, early, and late extrauterine growth retardation. To demonstrate relevant univariant associations,

Table 1. Characteristics of VLBW infants at birth and at 36 weeks' PMA

\begin{tabular}{lc}
\hline Parameter & VLBW infants $(n=95$ \\
\hline Sex, male, $N(\%)$ & $40(42)$ \\
AGA, $N(\%)$ & $67(71)$ \\
Gestational age (weeks) & $29 \pm 3$ \\
Birth & \\
Birth weight (g) & $1,060 \pm 256$ \\
$\quad$ SD score & $-0.84 \pm 0.68$ \\
Birth length (cm) & $35.8 \pm 3.6$ \\
SD score & $-0.41 \pm 1.01$ \\
Birth head circumference $(\mathrm{cm})$ & $25.6 \pm 2.4$ \\
SD score & $-0.40 \pm 0.77$
\end{tabular}

\section{Weeks PMA}

Weight SD score $\quad-1.90 \pm 0.61$

Length SD score $\quad-1.56 \pm 0.97$

Head circumference SD score $\quad-0.89 \pm 0.95$

AGA, appropriate for gestational age; PMA, postmenstrual age; VLBW, very low birth weight.

Data are expressed as mean \pm SD for continuous variables or $n(\%)$ for categorical variables. 


\section{Articles | Saenz de Pipaon et al.}

simple linear regression was used, examining leptin concentration against PFM and HOMA. Descriptive statistics are expressed as mean $( \pm \mathrm{SD})$ for continuous variables or $n(\%)$ for categorical variables.

\section{RESULTS}

Anthropometric data of the VLBW infants at birth and at 36 weeks' PMA are shown in Table 1. Catch-down growth, defined as a decrease in SD score $>0.67$, as stated by Ong et al. (22), was observed in the weight of 86 infants (92\%) and in the length of 77 infants (85\%) between birth and 36 weeks' PMA. Anthropometric data from 6 months' CA to 36 months of age are presented in Table 2 and Figure 1. Body weight, length, and head circumference increased over time $(P<0.001)$. Weight SD scores decreased and length SD scores increased from 6 months' CA to 36 months $(P<0.001)$. Catch-up growth, defined as a change in $z$-score $>0.67$ (ref. 16), was observed for weight in 18 (19\%) of the infants between 6 months' CA and 36 months. Head circumference $z$-score increased from 6 months' CA to 36 months $(P=0.006)$. In the follow-up study, $26 \%$ of infants were reported to be formula-fed from birth, $74 \%$ received human milk for an average of at least 9 weeks, whereas only

Table 2. Characteristics of VLBW infants from 6 months' CA to 36 months

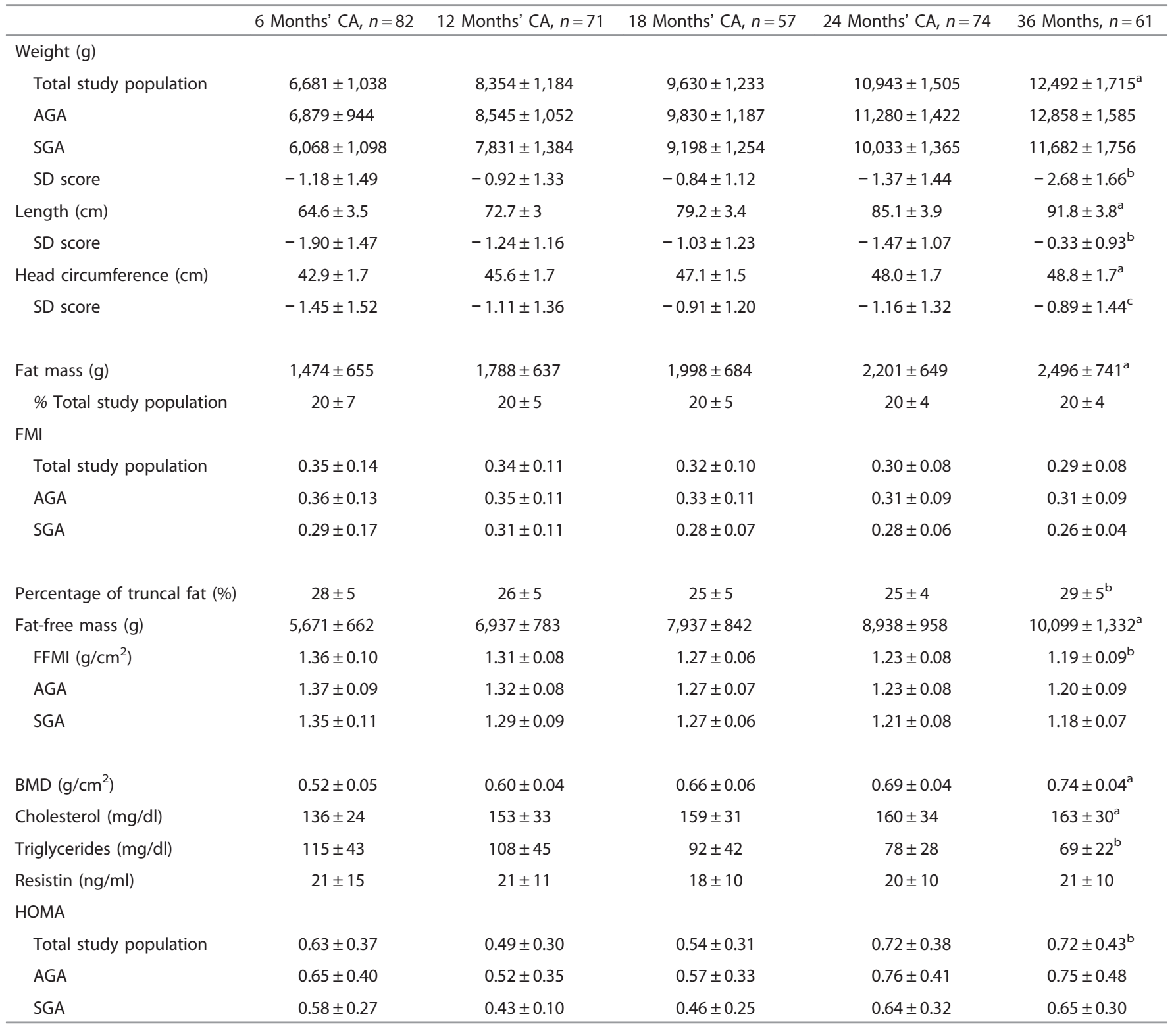

AGA, appropriate for gestational age; BMD, bone mineral density; CA, corrected age; FFMI, fat-free mass index; FMl, fat mass index; SGA, small for gestational age; VLBW, very low birth weight.

Data are expressed as mean \pm SD for continuous variables.

${ }^{a}$ Anthropometric indices, fat mass, fat-free mass, BMD, and cholesterol increased over time $(P<0.001)$.

'bignificant differences between 6 months' $C A$ and 36 months $(P<0.001)$.

'Significant differences between 6 months' CA and 36 months $(P=0.006)$. 


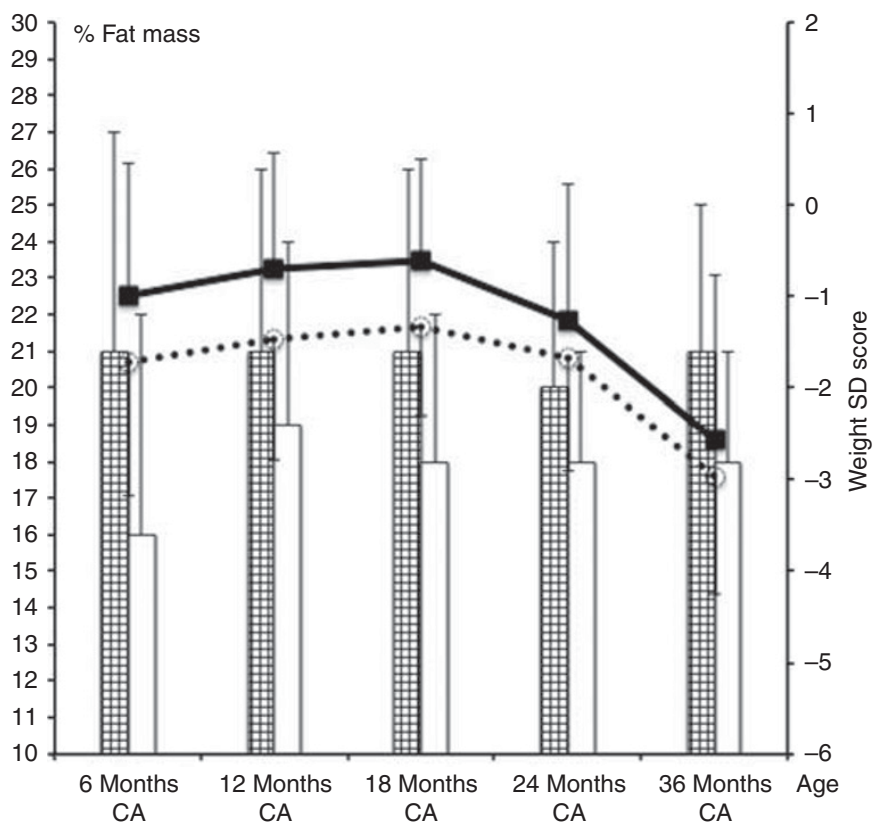

Figure 1. Weight SD score (lines) and percentage fat mass from 6 months' CA to 36 months (bars) in AGA (black, $n=71$ ) vs. small for gestational age (SGA) (open, $n=24$ ). Mixed-effect model repeat measurement analyses were performed. Percentage fat mass is significantly different between AGA and SGA from 6 months' CA to 36 months' $(P<0.001)$. Weight SD score does not differ significantly between groups. AGA, appropriate for gestational age; CA, corrected age.

14 infants were still receiving human milk at 3 months' CA (unpublished data).

FM, PFM, FMI, percentage trunk-FM, FFM, FFMI, and BMD are presented in Table 2 and Figure 1. FM, FFM, and BMD increased gradually from 6 months' CA to 36 months $(P<0.001)$. Whereas PFM remained stable $(P=0.714)$, FMI and FFMI decreased over time $(P=0.004$ and $P<0.001$, respectively). Percentage trunk-FM decreased from 6 to 18-24 months' $\mathrm{CA}$, and then increased at 36 months to values similar to those observed at 6 months' CA $(P<0.001$; Table 2). PFM and FMI were lower for SGA $(n=24)$ than for AGA infants $(n=71)$ from 6 months' CA to 36 months $(P<0.001)$.

Cholesterol, triglycerides, resistin, and HOMA-IR are presented in Table 2, and insulin-like growth factor I, leptin, and adiponectin are displayed in Figure 2. Cholesterol increased, particularly between 6 and 12 months' CA, whereas triglycerides steadily decreased during the study period $(P<0.001)$. Leptin decreased from 6 to 12 months' CA and then remained stable. Adiponectin decreased from 6 to 18 months' CA. No effects of time on resistin were observed. HOMA-IR decreased initially and then increased $(P<0.001)$. HOMA-IR was not statistically different between SGA and AGA infants throughout the study $(P=0.076)$.

PFM correlated positively with serum leptin concentrations throughout the study from 6 months' CA to 36 months $(r=0.444-0.583, P<0.001)$. HOMA-IR tended to be positively correlated with serum leptin concentration at 6 months' $\mathrm{CA}$, but this did not attain statistical significance $(r=0.303$; $P=0.061)$. HOMA, however, was positively associated with leptin at 12,18 , and 24 months' CA and 36 months $(r=0.341$,
$P=0.008 ; \quad r=0.356, \quad P=0.011 ; \quad r=0.345, \quad P=0.008 ;$ and $r=0.392, P=0.003$, respectively). The other biochemistries did not correlate consistently with PFM or HOMA-IR throughout the study period.

The effects of age, sex, being AGA vs. SGA status, and experiencing early and late extrauterine weight-SD score on PFM, FMI, FFMI, and HOMA-IR from 6 months' CA to 36 months are shown in Table 3. PFM and FMI were negatively associated with a decrease in weight-SD score from birth to 36 weeks' PMA (early extrauterine period; $P<0.001$ ) and from 36 weeks' PMA to 6 months' CA (late extrauterine period; $P=0.003$ ). FFMI was negatively associated with a decrease in early, but not late extrauterine weight-SD score. PFM $(+4.3 \pm 1.03 \%)$ and FMI $\left(+0.09 \pm 0.02 \mathrm{~g} / \mathrm{cm}^{2}\right)$ were higher in AGA than in SGA infants from 6 months' CA to 36 months. Overall, PFM and FMI did not differ by sex, but females had significantly less PFM than males had at 6 and 12 months' CA $(-2.80 \pm 1.36 \%(P=0.045)$ and $-2.89 \pm 1.29 \%$ $(P=0.028))$, respectively. FMI was lower in females than in males at 12 months' CA $\left(-0.06 \pm 0.03 \mathrm{~g} / \mathrm{cm}^{2}(P=0.037)\right)$. FFMI and $\operatorname{BMD}(P=0.001)$ were also lower in females than in males during the entire study period.

HOMA-IR was not significantly associated with early or late extrauterine $\Delta$ weight $\mathrm{SD}$ scores. Nor was HOMA-IR associated with PFM.

\section{DISCUSSION}

A decrease in weight-SD score before 6 months' CA was associated with a lower accretion of FM and FFM in VLBW preterm infants. Prenatal growth retardation and insufficient 

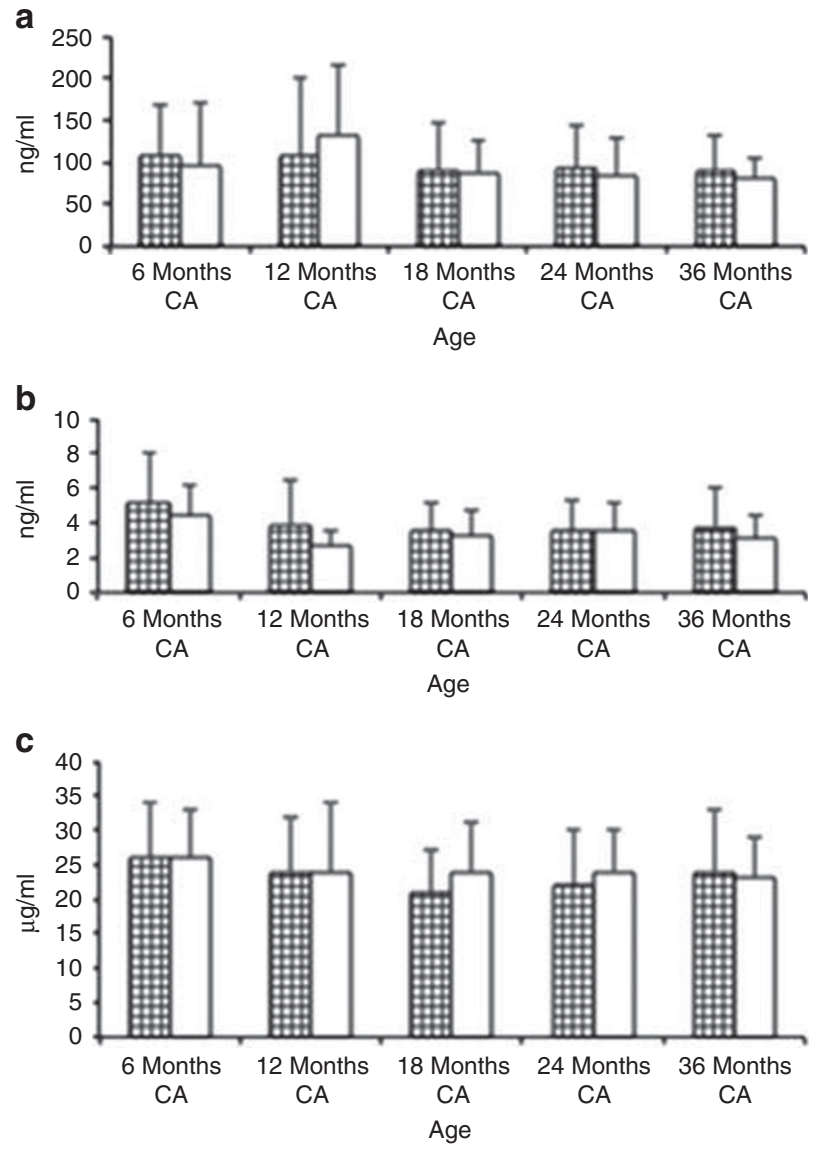

Figure 2. IGF-I, leptin and adiponectin serum concentrations. (a) IGF-I, (b) leptin, and (c) adiponectin of AGA (grid bars, $n=61$ ) and SGA (open bars, $n=20$ ) infants from 6 months' CA to 36 months. Mixed-effect model repeat measurement analyses were performed. No significant differences between groups were observed. AGA, appropriate for gestational age; CA, corrected age; IGF-I, insulin-like growth factor l; SGA, small for gestational age.

early/late extrauterine weight gain were associated with less FM from 6 months' CA to 36 months. A deficit in weight gain during the last trimester and in early postnatal life had a persistent effect on PFM and FMI through 36 months of age. We did not detect an effect of prenatal or postnatal growth on HOMA-IR in this cohort up to 36 months of age. Given the potential impact of poor growth during a relatively short time frame on later metabolic health, this study clarifies the effect of intrauterine and early postnatal growth on later body fatness and insulin resistance between 6 months' CA and 36 months in VLBW infants.

Growth and body composition were measured longitudinally from 6 months' CA to 36 months in a cohort of preterm infants whose protein and energy intakes approached recommended levels during the first few weeks of life (19). Prenatal and/or early postnatal growth retardation followed by catch-up growth between 6 months' CA and 36 months was not associated with the expected accretion of FM before 36 months. Although absolute FM and FFM increased from 6 months' CA to 36 months, PFM remained stable. Even the
Table 3. Estimate effects for fat mass percentage, fat mass index, fat-free mass index, and HOMA-IR between 6 months' corrected age and 36 months $^{\mathrm{a}}$

\begin{tabular}{|c|c|c|c|c|}
\hline $\begin{array}{l}\text { Dependent } \\
\text { variable }\end{array}$ & Parameter & Estimate & SE & Sig. \\
\hline \multirow{5}{*}{$\begin{array}{l}\text { Percentage fat } \\
\text { mass }\end{array}$} & AGA & 4.30 & 1.03 & $P<0.001$ \\
\hline & $\begin{array}{l}\text { Early extrauterine } \\
\text { weight gain }(\Delta-\text { SDS) }\end{array}$ & -2.34 & 0.70 & $P=0.001$ \\
\hline & $\begin{array}{l}\text { Late extrauterine weight } \\
\text { gain }(\Delta-\text { SDS })\end{array}$ & -1.02 & 0.34 & $P=0.003$ \\
\hline & Sex-female & - & - & - \\
\hline & Age & - & - & - \\
\hline \multirow[t]{9}{*}{ Fat mass index } & AGA & 0.09 & 0.02 & $P<0.001$ \\
\hline & $\begin{array}{l}\text { Early extrauterine } \\
\text { weight gain }(\Delta-\mathrm{SDS})\end{array}$ & -0.05 & 0.01 & $P<0.001$ \\
\hline & $\begin{array}{l}\text { Late extrauterine weight } \\
\text { gain }(\Delta-\text { SDS })\end{array}$ & -0.03 & 0.01 & $P<0.001$ \\
\hline & Sex-female & - & - & - \\
\hline & Age $=6$ months' $C A$ & 0.06 & 0.02 & $P=0.004$ \\
\hline & Age $=12$ months' $C A$ & 0.07 & 0.02 & $P=0.001$ \\
\hline & Age $=18$ months' CA & 0.03 & 0.01 & $P=0.066$ \\
\hline & Age $=24$ months' $C A$ & - & - & - \\
\hline & Age $=36$ months & - & - & - \\
\hline \multirow{9}{*}{$\begin{array}{l}\text { Fat-free mass } \\
\text { index }\end{array}$} & AGA & - & - & - \\
\hline & $\begin{array}{l}\text { Early extrauterine } \\
\text { weight gain }(\Delta-\mathrm{SDS})\end{array}$ & -0.02 & 0.01 & $P=0.032$ \\
\hline & $\begin{array}{l}\text { Late extrauterine weight } \\
\text { gain }(\Delta-\text { SDS })\end{array}$ & - & - & - \\
\hline & Sex-female & -0.04 & 0.01 & $P=0.002$ \\
\hline & Age $=6$ months' CA & 0.18 & 0.01 & $P<0.001$ \\
\hline & Age $=12$ months' $C A$ & 0.13 & 0.01 & $P<0.001$ \\
\hline & Age $=18$ months' $C A$ & 0.08 & 0.01 & $P<0.001$ \\
\hline & Age $=24$ months' $C A$ & 0.04 & 0.01 & $P=0.004$ \\
\hline & Age $=36$ months & - & - & - \\
\hline \multirow[t]{8}{*}{ HOMA-IR } & AGA & 0.11 & 0.06 & $P=0.076$ \\
\hline & $\begin{array}{l}\text { Early extrauterine } \\
\text { weight gain }(\Delta-\mathrm{SDS})\end{array}$ & 0.07 & 0.04 & $P=0.09$ \\
\hline & $\begin{array}{l}\text { Late extrauterine weight } \\
\text { gain }(\Delta-\text { SDS })\end{array}$ & - & - & - \\
\hline & Age $=6$ months' $C A$ & - & - & - \\
\hline & Age $=12$ months' $C A$ & -0.22 & 0.05 & $P<0.001$ \\
\hline & Age $=18$ months' $C A$ & -0.18 & 0.05 & $P=0.002$ \\
\hline & Age $=24$ months' $C A$ & - & - & - \\
\hline & Age $=36$ months & - & - & - \\
\hline
\end{tabular}

AGA, appropriate for gestational age; CA, corrected age; SGA, small for gestational age.

${ }^{a}$ Mixed-effect model repeat measurement analyses were performed to assess associations between prenatal (as a dichotomous variable, AGA vs. SGA), early and late extrauterine weight gain (both continuous variables, changes in SDS), and percentage fat mass, fat mass index, fat-free mass index, and HOMA-IR, from 6 to 36 months, using the MIXED procedure for SAS 9.1 software (SAS Institute). The models included AGA-SGA at birth, the age at evaluation $(6,12,18,24$, and 36 months), and early and late $\Delta-$ SD scores. Sex was used as a covariate. 
AGA infants had similar FM, not higher than term infants from 18 months' CA on, as would have been expected at term gestational age (9). Griffin and co-workers reviewed published data and concluded that preterm infants generally show less FM and FFM than term infants, as published by Butte et al. (23) in the first year (24). In contrast, Ramel et al. (25) showed higher FM in preterm infants compared with term infants at term age and similar FM at 4 months' CA. To our knowledge, our study is the first to evaluate the effect of prenatal and early growth of VLBW infants on later body composition up to 36 months of age.

Rapid postnatal growth in preterm infants from preterm birth up to term age, and in the first 3 months after term age, is supposedly harmful for development of body fat (13). Intrauterine and extrauterine growth retardation did not promote higher FM in our cohort possibly because no catchup occurred before 6 months' CA. In children, prematurity is associated with reduced body fatness. The findings of Fewtrell et al. related to reduced body fatness in 8-12 years of age in formerly premature children are particularly pertinent to the results presented here (13). As in our study, Fewtrell et al. (6) found a relationship between AGA at birth and insulin resistance and FM during the first years of life. This effect seems to be lost in adulthood (reviewed by Lapillonne and Griffin (26)). We found that the early and late extrauterine decrease in weight-SD score was associated with lower FM, and the early extrauterine decrease in weight-SD score was associated with lower FFM during the first 36 months of life. The use of FMI and FFMI, adjusted for height, allowed us to quantify the effect of growth independently on FM and FFM. No association was found in our study between adiposity and HOMA-IR, although a relationship was found between leptin and insulin resistance from 12 months' CA to 36 months. In formerly VLBW infants, insulin resistance may develop during childhood $(1,25)$ and adulthood, as assessed by fasted insulin concentrations or insulin resistance tests (reviewed by Lapillonne Griffin (26)). Most of the studies do not support a relationship between rapid weight gain in preterm infants during the first 18 months of life and later risk of insulin resistance. Weight gain from birth to 40 weeks' gestation is not associated with insulin resistance in children aged 4-10 years born prematurely (27). Growth in the first year of life did not affect glucose tolerance (14). Insulin concentrations at 9-12 years were associated with an increase in weight from 18 months to the age of measurement, but not with weight gain before 18 months (6). In adults born at term, there was no direct effect of postnatal weight velocity ( $0-4$ months) on adult HOMA-IR; however, weight velocity from 0 to 24 months positively predicted HOMA-IR (28). In SGA infants, the percentage of body fat in adulthood explained the differences in insulin sensitivity of those with rapid weight gain in the first 3 months of life (29). Our study showed no association between early and late extrauterine growth and insulin resistance, suggesting that growth retardation during initial hospitalization and after discharge did not affect HOMA-IR up to 36 months of age. Caution, however, must be taken in interpreting our results, given that not all the parents of the infants born during the recruitment period consented to participation in the study and only a small percentage of infants experienced significant catch-up growth. The effects of the study design were already considered previously (19); however, clinical characteristics presented here are representative of our NICU population. Parents of the infants included in our study received nutritional recommendations, but they are not obliged to follow a strict feeding protocol and our short-term follow-up.

In conclusion, our study indicates that reduced weight gain during gestation and the first few months of life has a significant impact on FM from 6 months' CA to 36 months in VLBW infants. Furthermore, early postnatal growth retardation does not influence insulin sensitivity. Postnatal growth retardation is a frequent clinical feature in VLBW infants. Our data do not support the hypothesis that interventions aimed at discouraging weight gain during hospitalization and after discharge would improve body composition or insulin resistance in preterm infants.

\section{STATEMENT OF FINANCIAL SUPPORT}

This study was partially funded by the Fondo de Investigación Sanitaria and Fondos FEDER (Grants: PI041631 to M.S.P. and PI100747 and PI1302195 to J.A.).

Disclosure: The authors declare no conflict of interest.

\section{REFERENCES}

1. Hofman PL, Regan F, Jackson WE, et al. Premature birth and later insulin resistance. N Engl J Med 2004;351:2179-86.

2. Tinnion R, Gillone J, Cheetham T, Embleton N. Preterm birth and subsequent insulin sensitivity: a systematic review. Arch Dis Child 2014;99:362-8.

3. Singhal A, Fewtrell M, Cole TJ, Lucas A. Low nutrient intake and early growth for later insulin resistance in adolecents born preterm. Lancet 2003;361:1089-97.

4. Bazaes RA, Alegría A, Pittaluga E, Avila A, Iñiguez G, Mericq V. Determinants of insulin sensitivity and secretion in very-low-birth-weight children. J Clin Endocrinol Metab 2004;89:1267-72.

5. Finken MJ, Kejizer-Veen MG, Dekker FW, et al. Preterm birth and later insulin resitance: effects of birth weight and postnatal growth in a population based longitudinal study from birth into adult life. Diabetologia 2006;49:478-85.

6. Fewtrell MS, Doherty C, Cole TJ, Stafford M, Hales CN, Lucas A. Effects of size at birth, gestational age and early growth in preterm infants on glucose and insulin concentrations at 9-12 years. Diabetologia 2000;43: 714-7.

7. Irving RJ, Belton NR, Elton RA, Walker BR. Adult cardiovascular risk factors in premature babies. Lancet 2000;355:2135-6.

8. Yanni D, Darendeliler F, Bas F, Aydin BK, Coban A, Ince Z. The role of leptin, soluble leptin receptor, adiponectin and visfatin in insulin sensitivity in preterm born children in prepubertal stages. Cytokine 2013;64:448-53.

9. Johnson MJ, Wootton SA, Leaf AA, Jackson AA. Preterm birth and body composition at term equivalent age: a systematic review and metaanalysis. Pediatrics 2012;130:e640-9.

10. Sepúlveda C, Urquidi C, Pittaluga E, et al. Differences in body composition and resting energy expenditure in childhood in preterm children born with very low birth weight. Horm Res Paediatr 2013;79: $347-55$. 


\section{Articles | Saenz de Pipaon et al.}

11. Breukhoven PE, Kerkhof GF, Willemsen RH, Hokken-Koelega AC. Fat mass and lipid profile in young adults born preterm. J Clin Endocrinol Metab 2012;97:1294-302.

12. Euser AM, Finken MJ, Kejizer-Veen MG, Hille ET, Wit JM, Dekker FW. Dutch POPS-19 Collaborative Study Group. Associations between prenatal and infancy weight gain and BMI, fat mass, and fat distribution in young adulthood: a prospective cohort study in males and females born very preterm. Am J Clin Nutr 2005;81:480-7.

13. Fewtrell MS, Lucas A, Cole TJ, Wells JC. Prematurity and reduced body fatness at 8-12 y of age. Am J Clin Nutr 2004;80:436-0.

14. Kerkhof GF, Willemsen RH, Leunissen RW, Breukhoven PE, Hokken-Koelega AC. Health profile of young adults born preterm: negative effects of rapid weight gain in early life. J Clin Endocrinol Metab 2012;97:4498-506.

15. Gray IP, Cooper PA, Cory BJ, Toman M, Crowther NJ. The intrauterine environment is a strong determinant of glucose tolerance during the neonatal period, even in prematurity. J Clin Endocrinol Metab 2002;87:4252-6.

16. Leipälä JA, Raivio KO, Sarnesto A, Panteleon A, Fellman V. Intrauterine growthrestriction and postnatal steroid treatment effects on insulin sensitivity in preterm neonates. J Pediatr 2002;141:472-6.

17. Rotteveel J, van Weissenbruch MM, Twisk JWR, Delemarre-Van de Waal HA. Infant and childhood growth patterns, insulin sensitivity, and blood pressure in prematurely born young adults. Pediatrics 2008;122:313-21.

18. Martos-Moreno GA, Barrios V, Sáenz de Pipaón M, et al. Influence of prematurity and growth restriction on the adipokine profile, IGF1, and ghrelin levels in cord blood: relationship with glucose metabolism. Eur J Endocrinol 2009;161:381-9.

19. Sáenz de Pipaón M, Martínez-Biarge M, Dorronsoro I, et al. Growth in preterm infants until 36 weeks postmenstrual age is close to target recommendations. Neonatology 2014;106:30-6.
20. Fenton R Tanis, Kim H Jae. A systematic review and meta-analysis to revise the Fenton growth chart for preterm infants. BMC Pediatr 2013; 13:59.

21. Rawlings DJ, Cooke RJ, McCormick K, et al. Body composition of preterm infants during infancy. Arch Dis Child Fetal Neonatal Ed 1999;80:F188-91.

22. Ong KK, Ahmed ML, Emmett PM, Preece MA, Dunger DB. Association between postnatal catch-up growth and obesity in childhood: prospective cohort study. Br Med J 2000;320:967-71.

23. Butte NF, Hopkinson JM, Wong WW, Smith EO, Ellis KJ. Body composition during the first 2 years of life: an updated reference. Pediatr Res 2000;47:578-85.

24. Griffin IJ, Cooke RJ. Development of whole body adiposity in preterm infants. Early Hum Dev 2012;88 (Suppl 1): S19-24.

25. Ramel SE, Gray HL, Ode KL, Younge N, Georgieff MK, Demerath EW. Body composition changes in preterm infants following hospital discharge: comparison with term infants. J Pediatr Gastroenterol Nutr 2011;53:333-8.

26. Lapillonne A, Griffin IJ. Feeding preterm infants today for later metabolic and cardiovascular outcomes. J Pediatr 2013;162:S7-16.

27. Regan FM, Cutfield WS, Jefferies C, Robinson E, Hofman PL. The impact of early nutrition in premature infants on later childhood insulin sensitivity and growth. Pediatrics 2006;118:1943-9.

28. Slining MM, Kuzawa CW, Mayer-Davis EJ, Adair LS. Evaluating the indirect effect of infant weight velocity on insulin resistance in young adulthood: a birth cohort study from the Philippines. Am J Epidemiol 2011;173:640-8.

29. Leunissen RW, Kerkhof GF, Stijnen T, Hokken-Koelega A. Timing and tempo of first-year rapid growth in relation to cardiovascular and metabolic risk profile in early adulthood. JAMA 2009;301:2234-42. 